\title{
Beitrag zur Kenntnis des Abbaus der Proteine im Darmkanal.
}

Von

\author{
Emil Abderhalden und Friedrich Kramm.
}

(Aus dem physiologischen Institute der Universität Halle a. S.)

(Der Redaktion zugegangen am 28. Februar 1912.)

Bei der Untersuchung des Darminhaltes werden stets nach Eiweißfütterung Aminosäuren neben Peptonen und Polypeptiden aufgefunden. Wie jüngst gezeigt werden konnte, ${ }^{1}$ ) ist die Menge der Aminosäuren eine ganz beträchtliche, jedoch überwiegen die Peptone stets. Dieser Befund läßt eine verschiedene Deutung des Umfanges des Abbaus der Proteine und Peptone im Darmkanal zu. Man kann einmal annehmen, daß in der Hauptsache Peptone zur Resorption gelangen. Mit ebensoviel Recht kann man jedoch auch den Schluß ziehen, daß der Abbau der Eiweißkörper im Darmkanal im wesentlichen zu Aminosäuren führt. Die Peptone überwiegen deshalb an Menge gegenüber den Aminosäuren, weil die letzteren rasch zur Resorption gelangen, während die Peptone ihres weiteren Abbaus durch die Fermente des Magendarmkanals harren.

Gegen die Möglichkeit eines vollständigen Abbaus der Proteine bis zu Aminosäuren im Darmkanal ist eingewandt worden, daß der Abbau der Eiweißkörper im Reagenzglas durch Pankreas- und Darmsaft auffallend langsam erfolgt. Der eine von uns hat bereits gemeinsam mit Gigon ${ }^{1}$ ) bei der hydrolytischen Spaltung von Polypeptiden den verzögernden Einfluß der Spaltprodukte zeigen können. Im Darmkanal werden die hemmenden Abbaustufen fortwährend durch Resorption beseitigt, während im Reagenzglas die entstehenden Produkte alle liegen bleiben. Es braucht dieser Umstand jedoch nicht der einzige Grund für den langsameren Abbau im Reagenzglas zu sein.

1) Emil Abderhalden, Über den Gehalt des Darminhaltes einiger Säugetiere an freien Aminosäuren, Diese Zeitschrift, Bd. 74, S. 436, 1911.

1) Emil Abderhalden und Alfred Gigon, Weiterer Beitrag zur Kenntnis des Verlaufs der fermentativen Polypeptidspaltung, Diese Zeitschrift, Bd. 53, S. 251, 1907.

Hoppe-Seyler's Zeitschrift f. physiol. Chemie. LXXVII. 
Wir sind zurzeit nicht in der Lage, ein genaues Bild über die Bedingungen des Abbaus der Nahrungsstoffe im Magendarmkanal zu geben. Einmal haben wir eine beständige Sekretion von Magensaft, Galle, Pankreas- und Darmsaft. Beständig wird neuer Chymus aus dem Magen in den Darmkanal in kleinen Portionen übergeführt. Dieser breitet sich in dünner Schicht auf einer großen Oberfläche aus und unterliegt nun der Einwirkung eines Gemisches von Verdauungssekreten unter ganz bestimmten vielleicht in bestimmter Weise wechselnden Bedingungen. Beim Abbau der Eiweißkörper entstehen saure und basisch reagierende Abbaustufen. Wahrscheinlich wird im Darmkanal von Fall zu Fall je nach der Reaktion des Verdauungsgemisches ein Ausgleich geschaffen, sodaß die Reaktion an Ort und Stelle stets in feinster Weise abgestuft wird. Es spricht auch vieles dafür, daß für verschiedene Abbaustufen verschiedene Fermente vorhanden sind. Solange wir alle diese Verhältnisse nicht genauer überblicken können, sind wir auch nicht in der Lage, beim Abbau der Eiweißkörper im Reagenzglas Bedingungen zu schaffen, die der Verdauung unter normalen Verhältnissen nahe kommen oder gar entsprechen.

Wir haben uns nun die Frage vorgelegt, ob der Abbau der Peptone des Darminhaltes nach dessen Entnahme aus dem Darme rasch weiter schreitet, wenn der Chymus bei $37^{\circ}$ aufbewahrt wird. Vorversuche zeigten, daß eine Verdünnung des Darminhaltes mit Wasser sofort für die weitere Verdauung im allgemeinen ungünstige Bedingungen schafft. Ebenso ergab der Zusatz von antiseptischen Stoffen, wie Toluol, Chloroform usw., Störungen, besonders wenn solche Mengen davon zugesetzt wurden, die einen Einfluß auf das Wachstum der Bakterien hatten. Wir haben nach diesen Erfahrungen den Chymus ohne jeden Zusatz und ohne ihn zu verdünnen in einem geschlossenen Gefäße bestimmte Zeiten im Brutschrank aufbewahrt. Wir bestimmten dann einmal den Gesamtstickstoffgehalt (Methode nach Kjeldahl), ferner den Ammoniakstickstoff (Methode KrügerReich-Schittenhelm) und endlich den Aminostickstoff (Methode van Slyke). Bei der Bestimmung des Aminostickstoffs nach van Slyke dauerte die Zersetzung in den einen Fällen 
genau 5, in anderen 10 Minuten. Um zu wissen, wieviel Aminostickstoff und Ammoniakstickstoff beim vollständigen Abbau der Peptone zum Vorschein kommt, haben wir einen Teil des Chymus jedesmal mit der dreifachen Menge rauchender Salzsäure 6 Stunden am Rückflußkühler gekocht. Dann wurde mit Natronlauge neutralisiert und mit den angegebenen Methoden Gesamtstickstoff, Ammoniakstickstoff und Aminostickstoff bestimmt.

Was die Bestimmung des Aminostickstoffs anbetrifft, so war es nicht möglich, den Darminhalt direkt zu untersuchen, weil die im Chymus vorhandenen gröberen Bestandteile Störungen verursachen. Wir haben deshalb den Chymus mit einer bestimmten Menge Wasser aufgekocht und dann filtriert. Der Filterrückstand wurde mit heißem Wasser gewaschen und die gesamten Filtrate in einem Maßkolben auf ein bestimmtes Volumen aufgefüllt. $\mathrm{Zu}$ den einzelnen Bestimmungen wurden dann aliquote Teile der Gesamtflüssigkeit verwendet. Unter den gegebenen Bedingungen haben die Ammoniakstickstoffwerte eine nur relative Bedeutung, weil beim Aufkochen die Möglichkeit gegeben ist, daß etwas Ammoniak entweicht. Wir hätten durch Zugabe von Säure zu dem Gemisch diese Möglichkeit ausschalten können. Es ist jedoch erfahrungsgemäß schwierig, solche Gemische genau zu neutralisieren. Da es uns in erster Linie auf die Aminostickstoffwerte ankam, mußten wir jede Möglichkeit einer sekundären Hydrolyse ausschalten. Wir kochten ganz kurz auf und führten alle Operationen sehr rasch durch. Es ist selbstverständlich auch die Möglichkeit vorhanden, daß bei der Bestimmung des Aminostickstoffs nach van Slyke eine Hydrolyse von besonders leicht spaltbaren Peptonen eintritt. Die Erfahrungen bei der Aminostickstoffbestimmung bei Polypeptiden zeigt zwar, daß die Gefahr einer Spaltung keine sehr große ist, doch liegen vorläufig über das Verhalten von langen Ketten von Aminosäuren noch keine genügenden Erfahrungen vor. Vergleicht man in den unten mitgeteilten Versuchen die bei 5 Minuten dauernder Zersetzung erhaltenen Werte mit denen, die nach 10 Minuten gefunden wurden, dann ergibt sich ohne weiteres, daß die Dauer der Einwirkung der salpetrigen Säure von großem Einfluß auf die Resultate ist. Es ist nicht mög- 
lich, a priori zu entscheiden, ob die 5 Minuten dauernde Einwirkung der salpetrigen Säure in allem Fällen genügt, oder ob nicht vielmehr 10 Minuten erforderlich sind, um die wirklichen Aminostickstoffwerte zu erhalten. Vergleichende Untersuchungen mit der Formoltitration nach Sörensen ergaben etwas höhere Werte, als wir sie bei der 5 Minuten langen Einwirkung der salpetrigen Säure nach van Slyke erhalten hatten. Die nach 10 Minuten dauernder Zersetzung erhaltenen Zahlen für den Aminostickstoff sind zu horch. Unsere Erfahrung zeigt, daß man die van Slykesche Methode von Fall zu Fall zu prüfen hat. Eine Vergleichung mit der Formoltitration nach Sörensen wird am raschesten ergeben, wie lange die Zersetzung dauern darf, ohne daß eine erhebliche Spaltung einsetzt. Die van Slykesche Methode hat gegenüber der Formoltitration den großen Vorzug der raschen Durchführbarkeit. Hat man die van Slykesche Methode mit Hillfe der Formoltitration für den einzelnen Fall eingestellt, dann leistet sie ohne $Z_{w}$ eifel Vorzügliches. Ohne die erwähnte Konttrolle ist die Deutung der Resultate, falls man absolute Werte zu erhalten wünscht. unsicher, besonders dann, wenn man unbekannte Gemische zu untersuchen hat. Wir haben die Fehler, die der Ammoniakund Aminostickstoff bestimmung nach der gegebenen Darlegung anhaften, durch Ausführung möglichst vieler Versuche zu kompensieren versucht. Wir betrachten die mitgeteilten Zahlen nicht als absolute Werte. Die Unterschiiede sind so beträchtliche, daß die gezogenen Schlußfolgerungen ohne Zweifel berechtigt sind.

Die folgende Zusammenstellung der erhaltenen Resultate zeigt, daß der Chymus kurz nach seiner Entnahme aus dem Dünndarm bereits erhebliche Mengen voin Aminostickstoff und auch von Ammoniak aufweist. Die erste Untersuchung des Darminhaltes erfolgte 20-30 Minuten mach erfolgter Tötung des Tieres. Untersucht wurde der Darminhalt vom Schwein. Nach einstündigem Aufbewahren im Brutschrank fand bereits eine erhebliche Zunahme des Aminosticksitoffs statt. Nach zweistündigem Verweilen bei $37^{\circ}$ war der Prozentsatz an Aminostickstoff weiter gestiegen. Nach 6 Stuniden war bereits etwa 
2/3 derjenigen Aminostickstoffmenge vorhanden, wie sie nach vollständiger Hydrolyse mit rauchender Salzsäure nachweisbar war. Unsere Beobachtungen sprechen ohne Zweifel dafür, daß im Darmkanal Bedingungen vorhanden sind, die einen raschen Abbau bis zu Aminosäuren gestatten. Wir zweifeln nicht daran, daß die Hydrolyse eine noch raschere sein würde, wenn die entstehenden einfachen Abbaustufen beseitigt werden könnten.

Gegen die erhobenen Befunde wird der Einwand erhoben werden, daß die Wirkung der Bakterien nicht ausgeschlossen war. Dagegen ist hervorzuheben, daß im Darmkanal die Bakterien ebenfalls ihre Wirksamkeit entfalten, und ferner ist darauf hinzuweisen, daß nach allen bisherigen Erfahrungen beim Abbau von Proteinen und Peptonen durch Bakterien eine erhebliche Desaminierung sich einstellt. Ein Blick auf die Ammoniakstickstoffwerte zeigt, daß diese nicht in gleichem Maße ansteigen, wie die Aminostickstoffmengen, ja in einzelnen Fällen blieb eine Vermehrung des Ammoniaks fast vollkommen aus.

Wir glauben unsere Beobachtungen in dem Sinne deuten zu können, daß im Darmkanal alle Bedingungen zu einer raschen Zerlegung der Eiweißkörper und Peptone bis zu Aminosäuren gegeben sind. Die erhaltenen Resultate stützen die Annahme, daß der Abbau der Eiweißkörper im Magendarmkanal ein sehr weitgehender bis vollständiger ist, $d$. h. bis $\mathrm{zu}$ den einfachsten Bausteinen, den Aminosäuren, führt.

a) Untersuchungen, bei denen die Zersetzung nach van Slyke 10 Minuten dauerte.

Versuch 1.

\begin{tabular}{|c|c|c|c|}
\hline & $\begin{array}{c}\text { 1. Probe } \\
\text { sofort untersucht }\end{array}$ & $\begin{array}{c}\text { 2. Probe } \\
\text { nach } 6 \text { Std. untersucht }\end{array}$ & $\begin{array}{l}\text { 3. Probe } \\
\text { Hydrolyse }\end{array}$ \\
\hline $\mathrm{N}$ als $\mathrm{NH}_{3}$ in $\mathrm{g}$. . . . & 0,1094 & 0,1712 & 0,2001 \\
\hline$\Rightarrow \mathrm{NH}_{2}>\gg . .$. & 0,2307 & 0,3884 & 0,4521 \\
\hline$>$ Gesamt- $N$ in $g$. & 0,6708 & 0,6777 & 0,7076 \\
\hline$>\quad \mathrm{NH}_{3}$ in $\%$ des Ges. $-\mathrm{N}$ & 16,31 & 25,26 & 28,27 \\
\hline$\Rightarrow, \mathrm{NH}_{2} \gg$, & 34,39 & 57,31 & 63,89 \\
\hline
\end{tabular}

Jede Probe enthält $70 \mathrm{~g}$ Darminhalt. 
Versuch 2.

\begin{tabular}{|c|c|c|c|}
\hline & $\begin{array}{c}\text { 1. Probe } \\
\text { sofort untersucht }\end{array}$ & $\begin{array}{c}\text { 2. Probe } \\
\text { nach } 6 \text { Std. untersucht }\end{array}$ & $\begin{array}{l}\text { 3. Probes } \\
\text { Hydrolysie }\end{array}$ \\
\hline $\mathrm{Nals} \mathrm{NH}_{3}$ in $\mathrm{g} . .$. & 0,028 & 0,0799 & 0,0927 \\
\hline$\gg \mathrm{NH}_{2} \gg \gg$. . . . & 0,0837 & 0,2311 & 0,2119 \\
\hline > Gesamt-N in $\mathbf{g}$. & 0,2102 & 0,3643 & 0,3306 \\
\hline$\triangleright \mathrm{NH}_{3}$ in $\%$ des Ges. $-\mathrm{N}$ & 13,32 & 21,91 & 28,03 \\
\hline$>>\mathrm{NH}_{2} \gg$, & 39,82 & 63,43 & 64,09 \\
\hline
\end{tabular}

Jede Probe enthält $90 \mathrm{~g}$ Darminhalt.

Versuch 3 .

\begin{tabular}{l|c|c|c}
\hline & $\begin{array}{c}\text { 1. Probe } \\
\text { sofort untersucht }\end{array}$ & $\begin{array}{c}\text { 2. Probe } \\
\text { nach 3 Std. untersucht }\end{array}$ & $\begin{array}{c}\text { 3. Probe: } \\
\text { Hydrolysse }\end{array}$ \\
\hline $\mathrm{Nals} \mathrm{NH}_{3}$ in g . . . . & 0,01541 & 0,02802 & 0,028022 \\
, $\mathrm{NH}_{2}$. . . . . & 0,0665 & 0,08858 & 0,1309 \\
, Gesamt-N in $\mathrm{g}$. & 0,1513 & 0,1373 & 0,1726 \\
, $\mathrm{NH}_{3}$ in $\%$ desGes.-N & 9,95 & 16,21 & 16,23 \\
, $\mathrm{NH}_{2}$, . & 42,95 & 64,52 & 75,84
\end{tabular}

Jede Probe enthält $50 \mathrm{~g}$ Darminhalt.

\section{Versuch 4 .}

\begin{tabular}{|c|c|c|c|}
\hline & $\begin{array}{c}\text { 1. Probe } \\
\text { sofort untersucht }\end{array}$ & $\begin{array}{c}\text { 2. Probe } \\
\text { nach } 3 \text { Std. untersucht }\end{array}$ & $\begin{array}{l}\text { 3. Probe } \\
\text { Hydrolysee }\end{array}$ \\
\hline $\mathrm{Nals} \mathrm{NH}_{3}$ in $\mathrm{g} . .$. & 0,03455 & 0,04735 & 0,7282 \\
\hline, $\mathrm{NH}_{2} \gg \gg . . .$. & 0,1562 & 0,23985 & 0,3539 \\
\hline , Gesamt-N in $\mathrm{g}$. . & 0,4145 & 0,4035 & 0,4780 \\
\hline$>\mathrm{NH}_{3}$ in $\%$ des Ges.-N & 8,34 & 11,74 & 15.23 \\
\hline$\Rightarrow \gg \mathrm{NH}_{2} \gg \gg \otimes$ & 37,68 & 60,81 & 74,02 \\
\hline
\end{tabular}

Jede Probe enthält $52 \mathrm{~g}$ Darminhalt. 
Versuch 5.

\begin{tabular}{|c|c|c|c|c|}
\hline & $\begin{array}{l}\text { 1. Probe } \\
\text { sofort } \\
\text { untersucht }\end{array}$ & $\begin{array}{l}\text { 2. Probe } \\
\text { nach 1 Std. } \\
\text { untersucht }\end{array}$ & $\begin{array}{l}\text { 3. Probe } \\
\text { nach 2 Std. } \\
\text { untersucht }\end{array}$ & $\begin{array}{l}\text { 4. Probe } \\
\text { Hydrolyse }\end{array}$ \\
\hline $\mathrm{N}$ als $\mathrm{NH}_{3}$ in $\mathrm{g}$. . & 0,03327 & 0,036776 & 0,038527 & 0,143 \\
\hline$\Rightarrow \mathrm{NH}_{2} \gg$ & 0,200875 & 0,28275 & 0,313125 & 0,4928 \\
\hline , Gesamt-N im $\mathbf{g}$ & 0,5499 & 0,5972 & 0,6164 & 0,6479 \\
\hline , $\mathrm{NH}_{3}$ in $\%$ des Ges.-N & 6,05 & 6,159 & 6,25 & 22,07 \\
\hline , \& $\mathrm{NH}_{2}$ > , & 36,53 & 47,34 & 50,80 & 76,04 \\
\hline
\end{tabular}

Jede Probe enthält $50 \mathrm{~g}$ Darminhalt.

Versuch 6.

\begin{tabular}{|c|c|c|c|c|}
\hline & $\begin{array}{l}\text { 1. Probe } \\
\text { sofort } \\
\text { untersucht }\end{array}$ & $\begin{array}{c}\text { 2. Probe } \\
\text { nach \& Std. } \\
\text { untersucht }\end{array}$ & $\begin{array}{c}\text { 3. Probe } \\
\text { nach 5 Std. } \\
\text { untersucht }\end{array}$ & $\begin{array}{l}\text { 4. Probe } \\
\text { Hydrolyse }\end{array}$ \\
\hline $\mathbf{N}$ als $\mathrm{NH}_{3}$ in $\mathbf{g} \cdot$. . . & 0,038527 & 0,06094 & 0,06144 & 0,105 \\
\hline$>>\mathrm{NH}_{2} \gg, \ldots$ & 0,143125 & 0,209 & 0,20975 & 0,293 \\
\hline , Gesamt-N $\mathbf{i n} \mathrm{g}$ & 0,36075 & 0,36075 & 0,36075 & 0,4378 \\
\hline$\Rightarrow \quad \mathrm{NH}_{3}$ in $\%$ ades Ges.- $\mathrm{N}$ & 10,68 & 16,89 & 17,03 & 23,98 \\
\hline$>, \mathrm{NH}_{2}>>$ & 39,66 & 57,92 & 58,15 & 66,92 \\
\hline
\end{tabular}

Jede Probe enthält $50 \mathrm{~g}$ Darminhalt.

\section{Versuch 7.}

\begin{tabular}{|c|c|c|c|}
\hline & $\begin{array}{l}\text { 1. Probe } \\
\text { sofort } \\
\text { untersucht }\end{array}$ & $\begin{array}{l}\text { 2. Probe } \\
\text { nach \& Std. } \\
\text { untersucht }\end{array}$ & $\begin{array}{c}\text { 3. Probe } \\
\text { nach } 5 \text { Std. } \\
\text { untersucht }\end{array}$ \\
\hline 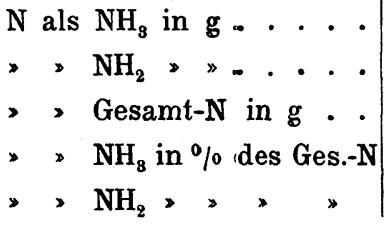 & $\begin{array}{l}0,025918 \\
0,09116 \\
0,26268 \\
9,867 \\
34,70\end{array}$ & $\begin{array}{l}0,07355 \\
0,17462 \\
0,3555 \\
20,69 \\
49,11\end{array}$ & $\begin{array}{l}0,0771 \\
0,1753 \\
0,3478 \\
22,15 \\
50,40\end{array}$ \\
\hline
\end{tabular}

Jede Probe enthält $40 \mathrm{~g}$ Darminhalt. 
Versuch 8.

\begin{tabular}{|c|c|c|c|c|}
\hline & $\begin{array}{l}\text { 1. Probe } \\
\text { sofort } \\
\text { untersucht }\end{array}$ & $\begin{array}{c}\text { 2. Probe } \\
\text { nach } 1 \text { Std. } \\
\text { untersucht }\end{array}$ & $\begin{array}{c}\text { 3. Probe } \\
\text { nach } 2 \text { Std. } \\
\text { untersucht }\end{array}$ & $\begin{array}{l}\text { 4. Probe } \\
\text { Hydrolyse }\end{array}$ \\
\hline $\mathrm{N}$ als $\mathrm{NH}_{3}$ in $\mathrm{g} . . .$. & 0,049035 & 0,05604 & 0,05954 & 0,0911 \\
\hline$\triangleright \mathrm{NH}_{2} \gg \gg . .$. & 0,126375 & 0,1478 & 0,162 & 0,3034 \\
\hline - Gesamt- $\mathrm{N}$ in $\mathrm{g}$. & 0,338 & 0,331 & 0,3292 & 0,4098 \\
\hline$\bowtie \mathrm{NH}_{3}$ in $\%$ des Ges. $\mathrm{N}$ & 14,50 & 16,93 & 18,08 & 22,22 \\
\hline$\triangleright \mathrm{NH}_{2} \gg \triangleright \gg$ & 37,40 & 44,65 & 49,20 & 74,03 \\
\hline
\end{tabular}

Jede Probe enthält $50 \mathrm{~g}$ Darminhalt.

Versuch 9.

\begin{tabular}{|c|c|c|c|c|}
\hline & $\begin{array}{l}\text { 1. Probe } \\
\text { sofort } \\
\text { untersucht }\end{array}$ & $\begin{array}{c}\text { 2. Probe } \\
\text { nach } 1 \text { Std. } \\
\text { untersucht }\end{array}$ & $\begin{array}{c}\text { 3. Probe } \\
\text { nach } 2 \text { Std. } \\
\text { untersucht }\end{array}$ & $\begin{array}{l}\text { 4. Probe } \\
\text { Hydrolyse }\end{array}$ \\
\hline $\mathrm{N}$ als $\mathrm{NH}_{3}$ in $\mathrm{g} \cdot \ldots$ & 0,049035 & 0,05564 & 0,05954 & 0,0911 \\
\hline$>\mathrm{NH}_{2} \gg \gg . . .$. & 0,126375 & 0,1478 & 0,162 & 0,3034 \\
\hline , Gesamt- $N$ in $\mathrm{g}$. & 0,338 & 0,331 & 0,3292 & 0,4098 \\
\hline$\Rightarrow \mathrm{NH}_{3}$ in $\%$ des Ges. $-\mathrm{N}$ & 14,50 & 16,93 & 18,08 & 22,22 \\
\hline$>, \mathrm{NH}_{2} \gg,>$ & $37, \mathbf{1 0}$ & 44,65 & 49,20 & 74,03 \\
\hline
\end{tabular}

b) Untersuchung, bei denen die Zersetzung nach van Slyke 5 Minuten dauerte.

Versuch 1.

\begin{tabular}{|c|c|c|}
\hline & $\begin{array}{l}\text { 1. Probe } \\
\text { sofort } \\
\text { untersucht }\end{array}$ & $\begin{array}{l}\text { 2. Probe } \\
\text { nach } 3 \text { Stunden } \\
\text { untersucht }\end{array}$ \\
\hline $\begin{array}{l}\mathrm{N} \text { als } \mathrm{NH}_{2} \text { in } \mathrm{g} . \ldots . . . \\
>\text { Gesamt-N in } \mathrm{g} \cdot \ldots . . \\
\Rightarrow \mathrm{NH}_{2} \text { in } \% \text { des Gesamt-N . . }\end{array}$ & $\begin{array}{l}0,0727 \\
0,2855 \\
25,48\end{array}$ & $\begin{array}{l}0,1069 \\
0,2995 \\
35,68\end{array}$ \\
\hline
\end{tabular}

Jede Probe enthält $50 \mathrm{~g}$ Darminhalt. 
Versuch 2.

\begin{tabular}{|c|c|c|}
\hline & $\begin{array}{l}\text { 1. Probe } \\
\text { sofort } \\
\text { untersucht }\end{array}$ & $\begin{array}{l}\text { 2. Probe } \\
\text { nach } 6 \text { Stunden } \\
\text { untersucht }\end{array}$ \\
\hline 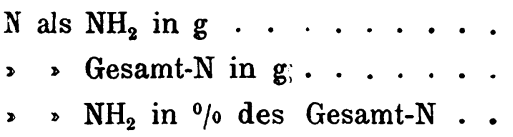 & $\begin{array}{l}0,0727 \\
0,2855 \\
25,48\end{array}$ & $\begin{array}{l}0,1258 \\
0,3012 \\
42,74\end{array}$ \\
\hline
\end{tabular}

$50 \mathrm{~g}$ D)arminhalt zu jeder Probe verwendet.

Versuch 3.

\begin{tabular}{|c|c|c|}
\hline & $\begin{array}{l}\text { 1. Probe } \\
\text { sofort } \\
\text { untersucht }\end{array}$ & $\begin{array}{l}\text { 2. Probe } \\
\text { nach } 3 \text { Stunden } \\
\text { untersucht }\end{array}$ \\
\hline 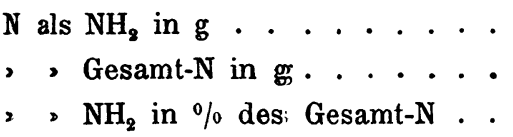 & $\begin{array}{l}0,0811 \\
0,3112 \\
26,06\end{array}$ & $\begin{array}{l}0,1295 \\
0,3215 \\
40,28\end{array}$ \\
\hline
\end{tabular}

$50 \mathrm{~g}$ Darminhalt zu jeder Probe verwendet.

Versuch 4 .

\begin{tabular}{|c|c|c|}
\hline & $\begin{array}{l}\text { 1. Probe } \\
\text { sofort } \\
\text { untersucht }\end{array}$ & $\begin{array}{l}\text { 2. Probe } \\
\text { nach } 6 \text { Stunden } \\
\text { untersucht }\end{array}$ \\
\hline 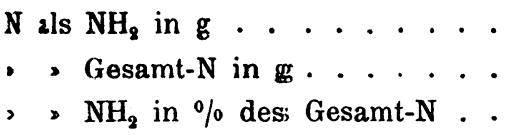 & $\begin{array}{l}0,0701 \\
0,28 \text { מ0 } \\
24,59\end{array}$ & $\begin{array}{l}0,1285 \\
0,2912 \\
44,13\end{array}$ \\
\hline
\end{tabular}

$50 \mathrm{~g}$ Darminhalt zu jeder Probe verwendet.

Versuch 5.

\begin{tabular}{|c|c|c|c|}
\hline & $\begin{array}{l}\text { 1. Probe } \\
\text { sofort } \\
\text { untersucht }\end{array}$ & $\begin{array}{c}\text { 2. Probe } \\
\text { nach 3 Stunden } \\
\text { untersucht }\end{array}$ & $\begin{array}{c}\text { 3. Probe } \\
\text { nach 6 Stunden } \\
\text { untersucht }\end{array}$ \\
\hline $\begin{array}{l}\mathrm{N} \text { als } \mathrm{NH}_{2} \text { in } \mathrm{g} \ldots \ldots \\
\Rightarrow \text { Gesamt-N in g . . } \\
\Rightarrow \mathrm{NH}_{2} \text { in } \% \text { des Ges.-N }\end{array}$ & $\begin{array}{l}0,1005 \\
0,4413 \\
22,79\end{array}$ & $\begin{array}{l}0,1416 \\
0,4553 \\
31,10\end{array}$ & $\begin{array}{c}0,1556 \\
0,4483 \\
34,70\end{array}$ \\
\hline
\end{tabular}

$50 \mathrm{~g}$ Darminhalt angewandt zu jeder Probe. 
434 Emil Abderhalden und Friedrich Kramm, Über Proteine

Versuch 6.

\begin{tabular}{c|c|c|c}
\hline & $\begin{array}{c}\text { 1. Probe } \\
\text { sofort } \\
\text { untersucht }\end{array}$ & $\begin{array}{c}\text { 2. Probe } \\
\text { nach 3 Stunden } \\
\text { untersucht }\end{array}$ & $\begin{array}{c}\text { 3. Probe } \\
\text { nach 6 Stuncen } \\
\text { untersucht }\end{array}$ \\
\hline $\mathrm{N}$ als $\mathrm{NH}_{2}$ in g . . . . & 0,0908 & 0,1137 & 0,1355 \\
> Gesamt-N in g . . . & 0,3362 & 0,3292 & 0,3362 \\
> $\mathrm{NH}_{2}$ in \% des Ges.-N & 27,00 & 34,54 & 40,30 \\
$50 \mathrm{~g}$ Darminhalt zu jeder Probe verwendet.
\end{tabular}

Um Mißverständnissen vorzubeugen, sei bemerkt, daß selbstverständlich die Zunahme an Aminostickstoff im Laufe der Verdauung nicht ohne weiteres mit der Bildung von Aminosäuren identifiziert werden darf, weil bei der Spaltung von Peptonen in einfachere Komplexe ebenfalls Aminogruppen zum Vorschein kommen können, indem $z$. B. eine säureamidartige Verkettung unter Wasseraufnahme gelöst wird. Wir dürfen anderseits unter Heranziehung direkter Bestimmungen der im Laufe der Verdauung sich bildenden Aminosäuren die benbachtete Zunahme an Aminostickstoff wenigstens zum Teil im Sinne einer Aminosäurebildung deuten. Der hohe Gehalt an Aminostickstoff nach sechsstündigem Verweilen im Brutschrank spricht ohne weiteres für die Bildung großer Mengen von Aminosäuren.

Die mitgeteilten Versuche werden im hiesigen Institut weiter fortgesetzt und auf ein verschiedenes Tiermaterial ausgedehnt. Ferner sind Versuche im Gange, die den Einfluß der Magenverdauung auf die Darmverdauung ergründen sollen. 